\title{
OPTICAL PHOTOMETRY OF BLACK-HOLE CANDIDATES
}

\author{
C. A. HASWELL
}

Columbia University

Dept. of Astronomy, 538 W. 120th St, New York, NY 10027

\begin{abstract}
.
The orbital light curves of SXTs are discussed. In principle, the orbital inclination can be determined from the ellipsoidal variations, allowing a complete solution for the component masses. Complications in this analysis are described, and the evidence for superhumps in the light curves is reviewed. Constraints on the compact-object masses derived from superhumps are described.
\end{abstract}

\section{Introduction}

This paper will discuss the soft X-ray transient (SXT) class of black-hole candidates which are introduced in the accompanying review by Charles. The long-term optical light curves of these objects form a fascinating topic (e.g., Callanan et al. 1995) which will not be discussed here; instead we will describe orbital and associated periodic photometric modulations, and the consequent system parameter determinations.

Quiescent light curves of SXTs show a double-humped 'ellipsoidal' orbital modulation due to the changing projected area of the Roche lobe filling mass donor. Maxima occur at quadratures, corresponding to the maximum projected area, and are hence expected to be symmetric. Minima occur at the orbital conjunctions; in general they have differing depths due to gravity darkening and heating by the disk. The amplitude of the ellipsoidal modulation depends on the orbital inclination $i$ of the system, so modelling of the observed ellipsoidal light curve can, in principle, constrain $i$, and hence contribute to an improved solution for the component masses.

There are several complications in the determination of $i$ from the ellipsoidal variations. Inevitably the light from the mass donor is diluted 
by the flux from the accretion disk; the extent of this diluting component can be assessed using high quality spectroscopic data (e.g., Marsh et al. 1994) but usually some uncertainties remain. Underestimating the diluting flux leads to an underestimate in the orbital inclination. The ellipsoidal light curve may be distorted by other variable contributions to the total light: the bright spot associated with the impact of the mass transfer stream on the edge of the disk (McClintock \& Remillard 1990); star spots on the secondary; and variable disk emission, including the "superhump" phenomenon.

Superhumps were first observed in superoutbursts of SU UMa stars, an extreme mass ratio subclass of CVs (Warner 1985). The superhump period, $P_{\text {sh }}$, can be expressed as

$$
P_{\mathrm{sh}}=(1+\epsilon) P_{\mathrm{orb}}
$$

where $P_{\text {orb }}$ is the orbital period, and $\epsilon$ typically has a value in the range 0.01-0.08 (Skillman \& Patterson 1993). There is an empirical relationship between the period excess, $\epsilon$, and the orbital period (Stoltz \& Schoembs 1984; Skillman \& Patterson 1993)

Theoretical work suggests that superhumps are due to tidal interactions in an extensive, non-circular, precessing disk (Whitehurst 1988; Hirose \& Osaki 1990). There are two prerequisites for superhumps. The first is a disk radius which exceeds the radius of the largest tidally stable particle orbit, the 'stability radius'. The disk can extend to the stability radius only for extreme mass ratios, hence the second prerequisite: an extreme mass ratio, $q \equiv M_{1} / M_{2} \gtrsim 4$, where $M_{1}$ is the compact object mass, and $M_{2}$ is the mass-donor star mass.

Theory is able to reproduce the empirical relationship between $\epsilon$ and $P_{\text {orb: }}$ Osaki (1985) derives

$$
\frac{P_{\text {orb }}}{P_{\text {prec }}}=\frac{3}{4} \frac{1}{\sqrt{q(1+q)}}\left(\frac{R_{\mathrm{d}}}{a}\right)^{3 / 2}
$$

where $P_{\text {prec }}$ is the disk precession period, $P_{\text {prec }}=\epsilon^{-1} P_{\mathrm{sh}}, R_{\mathrm{d}}$ is the radius of the disk, and $a$ is the binary separation. If either the disk radius or the mass ratio is measured (or assumed), the other quantity can thus be deduced from the period measurements; Mineshige et al. (1992) extend and elaborate on this and consequent constraints on the compact object mass.

Spectroscopic studies imply that SXTs have extreme mass ratios (see the contribution to these proceedings by Charles), so one requirement for a precessing non-circular disk is met in SXTs. Superhumps were detected in the outburst light curves of Nova Muscae 1991 and GS 2000+25 (Charles et al. 1991; Remillard et al. 1992; Bailyn 1992; see Sects. 3 and 4 for further 
discussion). Recently the detection of persistent superhumps in several extreme mass ratio CVs (Skillman \& Patterson 1993; Patterson et al. 1993; Patterson \& Skillman 1994) shows that an outburst state is not necessary for superhumps to occur. This implies that superhumps may need to be considered in the interpretation of the quiescent light curves of SXTs.

\section{A 0620-00: the Prototype and the Problems}

McClintock \& Remillard's (1986) seminal paper began the study of the ellipsoidal variations in quiescent SXT light curves, and foreshadowed some of the problems. Fig. 1a shows their BV band light curve, it agrees roughly with theoretical expectations, but the asymmetry between the two maxima requires an additional phase-variable contribution to the light curve. Further complications are apparent in subsequent observations: see Figs. 1b and 1c, Bartolini et al. (1990), and Haswell et al. (1993). Not only are the maxima asymmetric (Haswell 1992; Callanan 1993), but the sense of the asymmetry reverses; worse still, the same statement is true of the minima! In addition, the lower minima in Figs. $1 \mathrm{~b}$ and $1 \mathrm{c}$ appear deeper and sharper than a purely ellipsoidal curve would allow.

The anomalously deep minima in the quiescent light curves have been interpreted as grazing eclipses occurring when the disk radius is large (Karitskaya et al. 1988; Haswell et al. 1993). Hence both theoretical requirements for superhumps may be satisfied, and persistent superhumps might be expected. In CVs, the persistent superhumps typically have modest amplitudes, so by analogy persistent superhumps in SXTs might be expected to distort, rather than overwhelm, the ellipsoidal modulation in the orbital light curves. The orbital phase at which excess light due to the superhump is observed will depend upon the disk precession phase at which observations are made. Since the light curves shown in Fig. 1 result from averaging together data taken during many orbits, the superhump light would be effectively randomly distributed, and could account for the apparent secular variability in the shape of the orbital light curve (Haswell et al. 1993).

Haswell et al. (1993) attempted to deduce the orbital inclination from modelling of multi-colour light curves with ellipsoidal variations and a grazing eclipse, assuming a large circular disk; the lack of a deep minima at the opposite conjunction was interpreted as caused by low disk surface brightness. The data shown in Fig. 1c, however, makes this explanation unlikely: a drastic change in disk surface brightness would be required. As the cartoon in Fig. 2 shows, a non-circular disk leads to the possibility that grazing eclipses occur at one conjunction only. As the disk precesses, the grazing eclipse will occur first at one conjunction, then at the other. Hence the mo- 


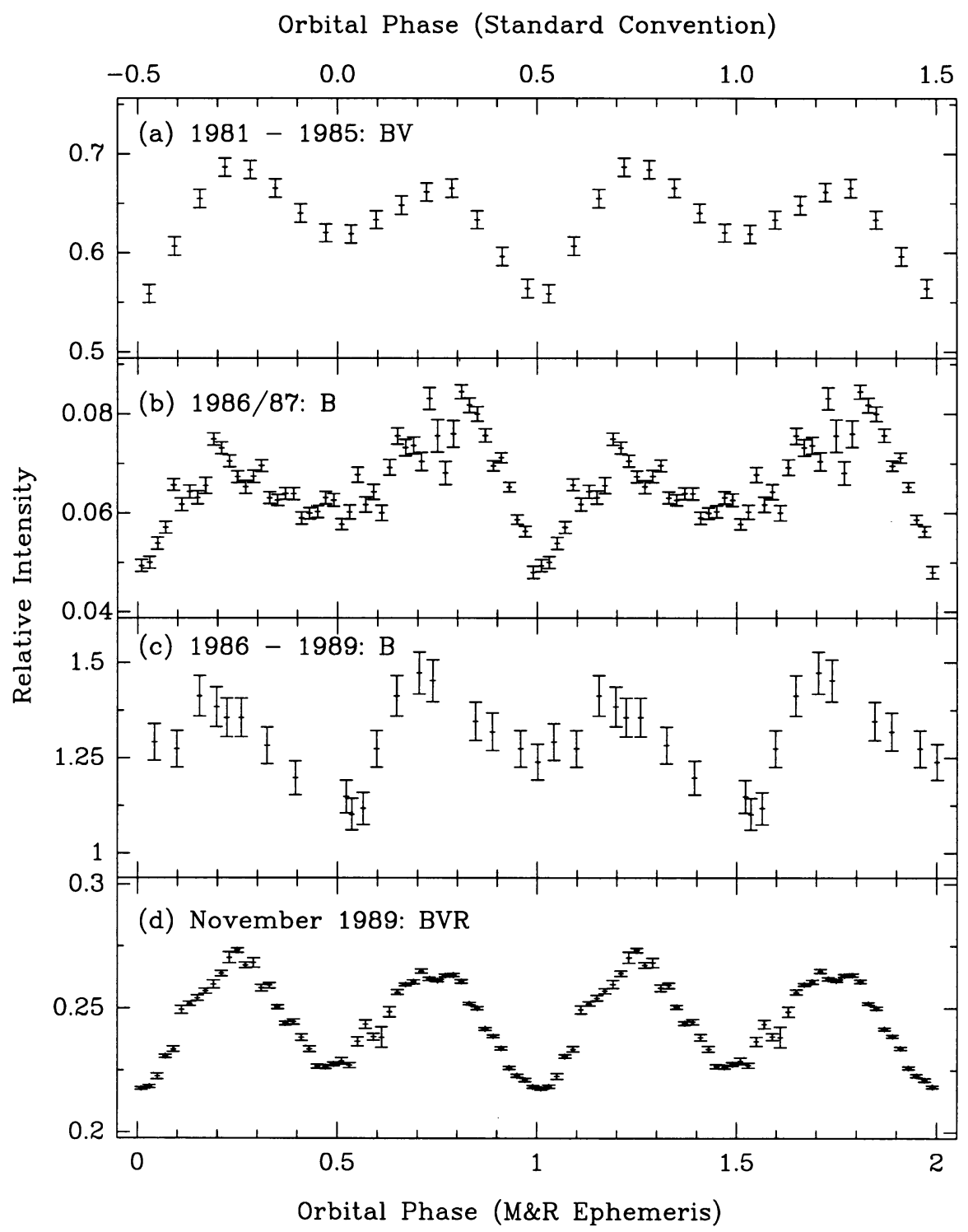

Figure 1. Orbital light curves of A0620-00: (a) from McClintock \& Remillard (1986); (b) from Haswell et al. 1993); (c) from Bartolini et al. 1990); (d) from Haswell (1992). Though the double-humped ellipsoidal variation is apparent, it is clearly contaminated. In the individual curves the maxima are distinctly asymmetric; the upper three curves show very prominent differences in the depths of the minima. There is, furthermore, definite secular variability in the shapes of the light curves: the asymmetry between the two maxima reverses between (a) and (b); the asymmetry between the minima reverses between (b) and (c); in (d) neither the maxima nor the minima appear so asymmetric. 


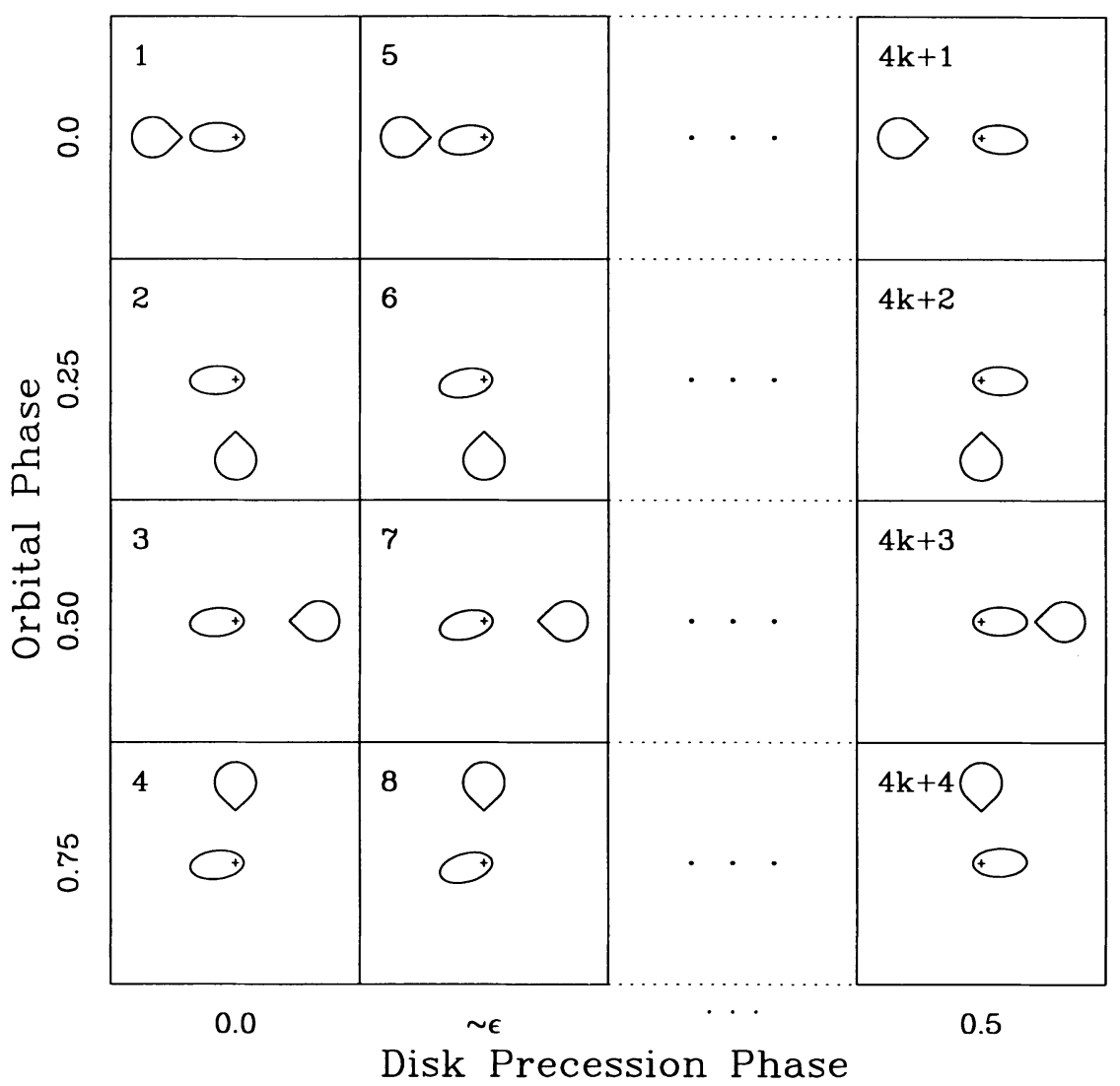

Figure 2. Cartoon depiction of the motion of the mass-donor and non-circular disk in a superhumping binary. Time increases down columns and from left to right, the sequence is indicated by the numbers in the upper left of each panel. Each column represents one binary orbit, and the total time elapsed is one half of the disk precession period. In the first panel the line of apsides of the disk is aligned with the mass-donor at binary phase 0.0 ; since the disk precession period is long compared to the orbital period, the line of apsides of the disk has moved only slightly by panel 5 . After many binary orbits have passed the disk's line of apsides has moved by 180 degrees. If $i$ is about 65 degrees then a grazing eclipse would be observed for the configuration shown in panel 1 (orbital phase 0.0 ) but not panel 3 (orbital phase 0.5 ). Conversely in the last column, a grazing eclipse might occur at orbital phase 0.5 but not orbital phase 0.0 .

del might simultaneously and self-consistently explain all the anomalies and secular changes shown in Fig. 1.

By November 1989 the photometric modulations were much closer to the expected ellipsoidal variation (see Fig. 1d). The maxima are no longer terribly asymmetric, and the difference between the depths of the minima is small enough to be accounted for by gravity darkening. This suggests that the disk shrank, so that neither grazing eclipses nor superhumps were 
distorting the light curve at this epoch. Spectroscopic work deduced a disk radius comparable to the size of the primary Roche lobe in 1986 (Johnston et al. 1989), by 1991 the disk radius was approximately half this size (Marsh et al. 1994; see also Orosz et al. 1994).

If the grazing-eclipse hypothesis is accepted, then it allows a simple geometrical constraint to be placed on the orbital inclination. It is reasonable to assume that the disk radius along the line of apsides is no larger than that of the largest coplanar circular disk which can be accommodated in the primary's Roche lobe. Adopting this prescription for the disk radius and demanding grazing eclipses be possible leads to a lower limit on the orbital inclination for a given mass ratio. Taking the mass ratio of $q=14.94$ from Marsh et al. (1994), we find a maximum disk radius $R_{\mathrm{d}}(\max )=0.63 a$. A system with this configuration will only exhibit grazing eclipses for $i>62^{\circ}$; this result is not strongly dependent on the exact value of $q$ until $q$ becomes much smaller than the preferred value. This limiting inclination changes by only $2^{\circ}$ for the range $10<q<20$.

The inclination derived from the grazing-eclipse constraint is in serious disagreement with that determined from the ellipsoidal variations in the $K$ band light curve, $31^{\circ}<i<54^{\circ}$ (Shahbaz et al. 1994a). The weakest part of their analysis is the assumption that the disk contributes negligible flux in the $\mathrm{K}$ band, which was justified by extrapolating the accretion disk flux in the optical assuming a power law spectrum. This extrapolation is uncertain, and Haswell (1992), Haswell et al. (1993) and Chevalier \& Mlovaisky (1993) show evidence for ellipsoidal amplitudes decreasing with increasing wavelength, contrary to the assertion that the flux becomes increasingly dominated by the mass-donor in the infrared. A direct measurement of the accretion disk flux from IR spectroscopy is desirable to resolve this issue.

\section{Nova Muscae 1991}

The quiescent orbital light curve of Nova Muscae 1991 shows a doublehumped ellipsoidal modulation with $P_{\text {orb }}=10.398(14)$ hours (Remillard et al. 1992). Again the ellipsoidal maxima are asymmetric, complicating the interpretation and making a reliable determination of $i$ from the ellipsoidal modulation difficult.

Nova Muscae 1991 showed a 10.5 hour modulation in its optical light curve during the decline from outburst (Bailyn 1992). This modulation is a likely superhump with $\epsilon \approx 0.014$ (Remillard et al. 1992). The error in the determination of $\epsilon$ is dominated by the uncertainty in $P_{\mathrm{sh}}$ : the data showed evidence for a change in $P_{\mathrm{sh}}$, but were insufficient to measure the period unequivocally at multiple epochs. This behaviour is consistent with a superhump interpretation: if $R_{\mathrm{d}}$ decreases then $P_{\text {prec }}$ will increase (Eq. 2), 
and $P_{\text {sh }}$ decreases. A lower limit for the compact object mass, $M_{1}$, can, however, be obtained using a formula derived from Eq. 2 above (Mineshige et al. 1992): $M_{1} \gtrsim 0.01 \epsilon^{-1} P_{\mathrm{orb}}(\mathrm{hr}) \mathrm{M}_{\odot}$. Adopting the longest candidate superhump period measured, (10.56(4) hours, see Bailyn 1992), leading to the lowest limit on $M_{1}$, we obtain

$$
M_{1} \gtrsim 6.66_{-1.30}^{+2.23} \mathrm{M}_{\odot}
$$

\section{GS $2000+25$}

In outburst, GS $2000+25$ exhibited an 8.33 hour photometric modulation (Charles et al. 1991); in quiescence, a shorter period was observed, indicative of ellipsoidal variations and an orbital period of $\sim 8.2$ hours (Callanan \& Charles 1991). These papers interpreted the outburst modulation as a superhump based on both the shape of the modulation and the period difference, though the latter was only a $3 \sigma$ result.

Subsequent observations of GS $2000+25$ reveal more complicated behaviour: Chevalier \& Ilovaisky (1993) determined a period of 8.25836 hours consistent with both their outburst and quiescent data. Evidence is also presented for a 10.015(2) hour period in quiescence, which is attributed to a 'distortion wave'. The beat period of the orbital and distortion wave modulations is 1.96 days; the orbital light curves show one shape for odd JD and another for even JD.

Reanalysing the same data, Shahbaz et al. (1994b) advocate an orbital period of 7.04 hours, dispensing with the distortion wave. However, Fig. 3 shows a high quality I band light curve of GS $2000+25$ (Haswell 1995) which strongly supports the 8.26 hour period; the 7.04 hour period is clearly inconsistent with these data.

The superhump and orbital periods can constrain the component masses in this system: $M_{1} \gtrsim 6 \mathrm{M}_{\odot}$ was obtained by Mineshige et al. (1992) using the periods reported by Charles et al. (1991) and Callanan \& Charles (1991).

\section{V404 Cyg}

V404 Cyg presents the best case for a stellar black hole, with a compact object mass exceeding $6.08 \pm 0.06 \mathrm{M}_{\odot}$ (Charles et al. 1994). Several groups have measured the ellipsoidal variations in the optical and IR light curves of this system. A best-fit inclination of $i=56^{\circ}$ and corresponding compact object mass of $12 \mathrm{M}_{\odot}$ is derived from the $\mathrm{K}$ band light curve (Shahbaz et al. 1994c), assuming a negligible contribution from the disk in that passband. This assumption is motivated by the measured contribution of the disk in 


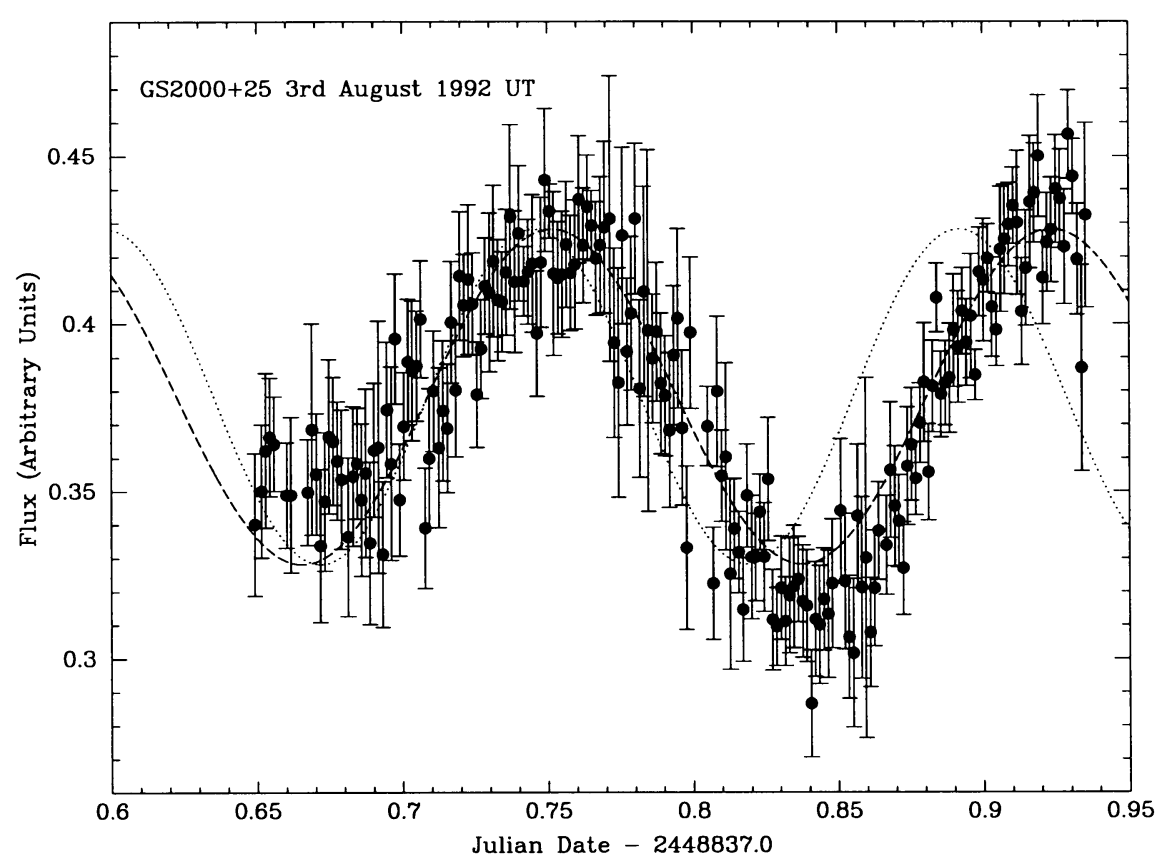

Figure 3. I band light curve of GS $2000+25$ taken at the McDonald Observatory 2.7 meter in excellent conditions. This single night of data clearly rules out the proposed 7.04 hour orbital period: a 3.52 hour sine wave is shown by the dotted line. The 8.26 hour period is strongly supported: the dashed line shows a 4.13 hour sine wave.

the optical, which is extrapolated into the IR. The extrapolation may be invalid as it predicts a disk contribution of $\sim 10 \%$ in the $\mathrm{R}$ band, while variability of amplitude $20 \%$ is observed superimposed upon the $R$ band ellipsoidal modulation (Pavlenko et al. 1994). This extra $\mathrm{R}$ band variability has a period of $\sim 6.2$ hours, and likely arises in the accretion flow. Variability superimposed on the ellipsoidal modulation is also observed in the I and $\mathrm{H}$ bands (Wagner et al. 1992; Robinson et al. 1994).

A further complication is apparent in the $\mathrm{H}$ band data: modelling the ellipsoidal light curve requires a source of X-ray heating of luminosity $\sim 310^{36} \mathrm{erg} \mathrm{s}^{-1}$, almost 400 times greater than that observed (Wagner et al. 1994). Independent of the complications, by adopting the most conservative set of assumptions to obtain an extreme lower limit on the orbital inclination, Robinson et al. (1994) deduce an upper limit of $M_{1}<12.0 \mathrm{M}_{\odot}$ for the mass of the compact object. 


\section{GRO J0422+32}

This object provides the latest opportunity to study the quiescent light curve of an SXT. A 5.1 hour period has been detected in the low-state photometry (Chevalier \& Mlovaisky 1994; Garcia et al. 1994; Callanan et al. 1995). It is not yet clear whether this modulation is orbital in origin, though there is some spectroscopic support for this idea (Harlaftis et al. 1994; Charles, these proceedings). The light curves are complex and changing, so it is highly unlikely that the variability is purely ellipsoidal.

If the orbital period were 5.1 hours, then a likely superhump was detected in November 1992 (Kato et al. 1992b), leading to a tentative mass estimate for the compact object (Kato et al. $1992 \mathrm{~b}$ ): $2.9 \lesssim M_{1} / \mathrm{M}_{\odot} \lesssim 6.2$.

\section{Conclusions}

The determination of orbital inclinations from modelling of ellipsoidal variations is complicated by other effects which distort the orbital light curves. While observations in the IR may better isolate the ellipsoidal component in the light curve, it is not clear that SXT disks produce negligible flux in the IR. The extensive data on A 0620-00 seem to suggest that the quiescent light curve becomes more purely ellipsoidal as time elapses since the outburst. Observations in late quiescence, with simultaneous spectroscopic determinations of the disk flux at the appropriate wavelength, hold the best promise for a robust determination of $i$.

Superhumps, while likely responsible for the contamination of the ellipsoidal modulation, offer an alternative approach to the determination of the system parameters in SXTs.

New SXTs are continually being discovered, providing an increasing number of systems to apply these techniques to. Eventually an SXT exhibiting X-ray eclipses will be found, yielding a precise determination of $i$, and hence providing the opportunity to assess the merits of the techniques discussed in this review.

Acknowledgements. Thanks to Paul Callanan, Phil Charles, Joe Patterson, and Rob Robinson for helpful discussions. I am also grateful to Paul Callanan for comments on this paper and providing data used in Fig. 1.

\section{References}

Bailyn, C.D. 1992, ApJ 391, 298

Bartolini, C. et al. 1990, in Structure and Emission Properties of Accretion Disks, IAU Colloq. No. 129, C. Bertout, S. Collin, J.-P. Lasota \& J. Tran Thanh Van (Eds.), Editions Frontières (Gif-sur-Yvette), p. 371 
Callanan, P.J. 1993, PASP 105, 961

Callanan P.J. \& Charles P.A. 1991, MNRAS 249, 573

Callanan, P.J. et al. 1995, ApJ (March 1 issue)

Casares, J. \& Charles, P.A. 1994, XXIInd IAU General Assembly Posters, H. van Woerden (Ed.), p. 83

Charles, P.A. 1995, these Proceedings

Charles, P.A. et al. 1991, MNRAS 249, 567

Chevalier, C. \& Movaisky, S.A. 1993, A\&A 269, 301

Chevalier, C. \& Ilovaisky, S.A. 1994, Haute-Provence Preprint No. 83

Garcia, M.R. et al. 1994, BAAS 25, 1381

Harlaftis, E. et al. 1994, in The Evolution of X-Ray Binaries, S.S. Holt \& C.S. Day (Eds.), AIP Conference Proc. Vol. 308, p. 91

Haswell, C.A. 1992, Ph.D. Thesis, University of Texas at Austin

Haswell, C.A. 1995, (in preparation)

Haswell, C.A. et al. 1993, ApJ 411, 802

Hirose, M. \& Osaki, Y. 1990, PASJ 42, 135

Johnston, H.M., Kulkarni, S.R. \& Oke, J.B. 1989, ApJ 345, 492

Karitskaya, E.A. \& Bochkarev, N.G. 1988, Perem. Zvezdy 22 (6), 943

Kato, T., Mineshige, S. \& Hirata, R. 1992a, IAU Circ. 5676

Kato, T., Mineshige, S. \& Hirata, R. 1992b, IAU Circ. 5704

Marsh, T.R., Robinson, E.L. \& Wood, J.H. 1994, MNRAS 266, 137

McClintock, J.E. \& Remillard, R.E. 1986, ApJ 308, 110

McClintock, J.E. \& Remillard, R.A. 1990, ApJ 350, 386

Mineshige, S., Hirose, M. \& Osaki, Y. 1992, PASJ 44, L15

Orosz, J.A. et al. 1994, ApJ (in press)

Osaki, Y. 1985, A\&A 144, 369

Patterson, J. \& Skillman, D.R. 1994, C.A.L. preprint number 551

Patterson, J. et al. 1993, ApJS 86, 235

Pavlenko, E.P. et al. 1994, XXIInd IAU General Assembly Posters, H. van Woerden (Ed.), p. 86

Remillard, R.A., McClintock, J.E. \& Bailyn, C.D. 1992, ApJ 399, L145

Robinson, E.L., Sanwal, D. \& Zhang, E. 1994, XXIInd IAU General Assembly Posters, H. van Woerden (Ed.), p. 87

Shahbaz T., Naylor T. \& Charles P.A. 1994a, MNRAS 268, 756

Shahbaz T. et al. 1994b, MNRAS 268, 763

Shahbaz, T. et al. 1994c, MNRAS (in press)

Skillman, D.R. \& Patterson, J. 1993, ApJ 417, 298

Stoltz, B. \& Schoembs, R. 1984, A\&A 132, 187

Wagner, R.M. et al. 1992, ApJ 401, L97

Wagner, R.M. et al. 1994, (preprint)

Warner, B. 1985, in Interacting Binaries, P.P. Eggleton \& J.E. Pringle (Eds.), Reidel Publishing Company, p. 367.

Whitehurst, R. 1988, MNRAS 232, 35

\section{Discussion}

D. O'Donoghue: MEM eclipse mapping of $\mathrm{Z}$ Cha (see O'Donoghue, 1990, MNRAS 246, 29) shows that the superhump light source has a bright feature along the line of centers, precisely where C. Haswell needs a light source to model her infrared light curve variations. 
W. Kundt: According to the little I know about superhumps in cataclysmic variables, an eccentric disk is not without problems. For instance, a $180^{\circ}$ phaseshift has been seen during transition from outburst to quiescence.

C. Haswell: I'm not familiar with the particular observations you are referring to ${ }^{1}$. As far as I know the precessing non-circular disk model has been spectacularly successful in explaining the observed properties of extreme mass ratio CVs. For example Patterson, Halpern \& Shambrook (1993, ApJ 419, 803) recently reported on spectroscopy of AM CVn in which the absorption lines from the disk show pronounced periodic variations in skewness. This feature is very successfully explained by a simple precessing noncircular disk model.

J. van Paradijs: Do you know of attempts to look for the beat between the orbital and super hump periods from the variation in the orbital phase at which the maximum in the perturbation occurs in the light curve, e.g., in the 15 year of data collected on A 0620-00.

C. Haswell: As far as I know this has not been done yet. Obviously it should be done using all the data collected by the many different observers who have studied A 0620-00. In addition to the data I showed, I learned at this conference from Paul Callanan that Jeff McCLintock has some unpublished data which shows a grazing eclipse feature. I suspect features have posed a challenge to interpretation. If the data are extensive enough, we may be able to follow the evolution of the precession period as the disk shrinks.

S.R. Kulkarni: If the secondary is indeed tidally locked, there should be tremendous tidal dissipation which should lead to great chromospheric activity. Is there any observational evidence for this (e.g., the Wilson-Bappu effect, spots, etc.)?

C. Haswell: One of the first explanations we considered for the photometric irregularities in A 0620-00 was migrating star spots on the mass donor (Haswell, Robinson \& Horne, 1990, in Accretion Powered Compact Binaries, C.W. Muche (Ed.), Cambridge University Press). We now have a body of evidence from A 0620-00 and other systems that a precessing non-circular disk contributes to the photometric irregularities. It is possible, however, that both these phenomena contaminate the light curves. If a precessing non-circular disk is included in the model it is difficult to identify unequivocal evidence for star spots.

\footnotetext{
${ }^{1}$ Note added: In his equation 62 , Osaki $(1985, A \& A 144,369)$ discusses this observation in VW Hyi, and gives a possible explanation within the context of the precessing noncircular disk model.
} 